\title{
Feasibility of multiple short, 40-s, intra-procedural ECG recordings to detect immediate changes in heart rate variability during catheter ablation for arrhythmias
}

\author{
Phang Boon Lim • Louisa C. Malcolme-Lawes • Thomas Stuber • Michael Koa-Wing • \\ Ian J. Wright • Therese Tillin • Richard Sutton • D. Wyn Davies • Nicholas S. Peters • \\ Darrel P. Francis • Prapa Kanagaratnam
}

Received: 12 January 2011 / Accepted: 14 April 2011 / Published online: 7 June 2011

(C) The Author(s) 2011. This article is published with open access at Springerlink.com

\begin{abstract}
Purpose This study aims to evaluate a method to detect heart rate variability (HRV) changes using short ECG segments during ablation for arrhythmias.

Methods HRV was averaged from sequentially shorter time windows from 5-min ECG recordings in 15 healthy volunteers. The 40 -s window was identified as the shortest duration that yielded reproducible values in high frequency (HF) and low frequency (LF) HRV. This method was validated in patients undergoing tilt table testing to see if the expected modulation in HRV that occurs prior to syncope could be detected from multiple 40-s recordings. Lastly, this method was used to assess HRV changes in 75 patients undergoing ablation for atrial fibrillation (AF) and other arrhythmias, to see if autonomic modulation as a result of ablation could be detected. A further 14 patients had stepwise HRV measurements at different stages of the AF ablation procedure to determine whether intraprocedural HRV changes could be detected.

Results HRV, averaged from multiple 40-s recordings, demonstrated the expected increase immediately preceding syncope compared with baseline (LF: $341 \pm 311-1,536 \pm$ $1,368 \mathrm{~ms}^{2}, p<0.05$; HF: $342 \pm 339-1,628 \pm 1,755 \mathrm{~ms}^{2}, p<$ $0.05)$. AF ablation, particularly following right pulmonary
\end{abstract}

PB Lim and LC Malcolme-Lawes are joint first authors.

P. B. Lim $(\bowtie) \cdot$ L. C. Malcolme-Lawes $\cdot$ T. Stuber $\cdot$

M. Koa-Wing • I. J. Wright • T. Tillin • R. Sutton • D. W. Davies •

N. S. Peters $\cdot$ D. P. Francis $\cdot$ P. Kanagaratnam

Cardiology Department,

Imperial College Healthcare NHS Trust,

St Mary's Hospital,

Praed Street,

London W2 1NY, UK

e-mail: pblim@imperial.ac.uk vein circumferential ablation, produced immediately detectable reductions in LF $\left(153 \pm 251-50+116 \mathrm{~ms}^{2}, p<0.001\right)$ and HF $\left(86 \pm 195-33 \pm 83 \mathrm{~ms}^{2}, p<0.001\right)$ without any change in RR interval $(877 \pm 191-843 \pm 220 \mathrm{~ms}, p=0.261)$. Ablation for atrial flutter did not change the mean RR interval, LF or HF HRV. Conclusion Averaging multiple 40-s windows give valid HF and LF HRV measurements that enable detection of intraprocedural changes. Left atrial ablation around the right-sided pulmonary veins is unique in producing reductions in HRV. This method has the potential for use as an endpoint marker for adjunctive autonomic ablation procedures.

Keywords Heart rate variability - Catheter ablation - Atrial fibrillation - Arrhythmias

\section{Introduction}

The intrinsic cardiac autonomic nervous system has been implicated in initiation and maintenance of atrial fibrillation (AF) by animal studies showing that autonomic stimulation leads to pulmonary vein ectopy [1] and increases susceptibility of atria to develop AF in the presence of ectopic activity [2].

In humans, modification of the autonomic nervous system has been suggested for prevention of AF [3, 4] by targeting presumed sites of dense atrial autonomic innervation [5]. Currently, there are no established techniques to monitor autonomic modification during ablation, so adjunctive autonomic ablation is performed either by empirical ablation at predetermined anatomical sites [6] or by targeting sites that cause atrioventricular block (AV) block [3, 4, 7, 8]. These are not ideal end-points because although they imply that autonomic ablation may have taken place, it is not possible to ascertain the extent of this autonomic modifica- 
tion in the context of the patient's overall cardiac autonomic function.

Heart rate variability (HRV), as an index of cardiac autonomic modulation, might have potential for such monitoring. Oscillations in the interval between consecutive heart beats can be measured on fairly short recordings [9] by applying spectral analysis to the RR interval series, yielding HF (the "high frequency" oscillations of respiratory sinus arrhythmia) and LF (the lower frequency intrinsic cardiac oscillations with a cycle time of approximately $10 \mathrm{~s}$ ) components of HRV. Autonomic ablation is known to attenuate LF and HF HRV. However, HRV has previously only been measured from recording times of many minutes or hours $[8,10,11]$, preventing real-time use for intraprocedural monitoring during catheter ablation for cardiac arrhythmias. The catheter laboratory is a more complex recording environment than an ambulant outpatient setting, with frequent ectopy caused by intracardiac catheters, which necessitates a technique of HRV assessments from ECG segments short enough to be ectopy-free for adequate HRV analysis, without unduly prolonging procedure duration.

In this study, we hypothesised that the effects of ablation procedures on HRV can be detected using multiple ECG segments short enough to permit real-time monitoring of autonomic modulation.

To test this, we first estimated the shortest ECG recording duration that still yielded reproducible average LF and HF HRV values in healthy volunteers. We then validated this technique by averaging HRV using multiple short-duration recordings in patients undergoing tilt table testing to see if the expected modulation in the autonomic nervous system that occurs prior to syncope could be detected using this method. Lastly, we used this method to assess HRV in patients undergoing ablation for AF and other arrhythmias or negative electrophysiological studies, to see if autonomic modulation as a result of ablation could be detected intra-procedurally.

\section{Methods}

\subsection{Validation of short recordings}

Fifteen healthy normal volunteers, who were not taking any drugs, were studied with ECG recordings (Hewlett-Packard 78351A) acquired from each subject for 20 min while lying still in a quiet room. The ECG was acquired from the limb lead with the largest $\mathrm{R}$ wave (usually lead II). Data was sampled at $1,000 \mathrm{~Hz}$ on a computer using an analogue-todigital converter (National Instruments) and stored for offline analysis of HRV using Matlab (Natick, MA, USA) [12]. RR intervals were determined and then converted into an evenly spaced temporal sequence at $5 \mathrm{~Hz}$. Having identified ectopic-free segments, autoregressive spectral analysis was then carried out on the complete segment [12]. Low frequency (LF: $0.04-0.15 \mathrm{~Hz}$ ) and high frequency (HF: $0.15-0.4 \mathrm{~Hz}$ ) spectral power components were then calculated from the autoregression profiles by integration [12].

A 5-min $(300 \mathrm{~s})$ recording segment free of ectopy was selected for LF and HF HRV analysis. Further sampling of progressively shorter segments $(2 \times 150,4 \times 75,6 \times 50,8 \times 40$, $10 \times 30$, and $15 \times 20 \mathrm{~s}$ segments) of data within the 5 -min recording was selected for HRV analysis to ascertain the shortest recording window that yielded reproducible average LF and HF values. The HRV indices for each of the recording durations were compared by repeated measures analysis of variance (ANOVA) with Newman-Keuls correction for comparison between the means. The HRV indices were further tested in random effects ANOVA and variance components models and intra-class correlation coefficients were calculated.

Further validation of the technique was conducted on ten patients undergoing tilt table testing for vasovagal syncope. LF and HF HRV were averaged from multiple 40-s ECG recordings within a 5-min window at the beginning of the tilt, and immediately preceding syncope to determine if the known changes in autonomic modulation that occur before syncope [13] were detected adequately using this technique.

\subsection{Heart rate variability from short recordings} during electrophysiological study and ablation

We studied 75 patients with symptomatic palpitations referred to our institution for electrophysiological (EP) study and ablation to see if the expected HRV changes could be identified following ablation. All patients gave full written informed consent for the procedure in-line with local institutional policy. There were 15 patients in each ablation arm, undergoing ablation for paroxysmal AF, atrial flutter, AV nodal re-entrant tachycardia (AVNRT) and AV re-entrant tachycardia (AVRT; Table 1). Fifteen patients had a negative EP study without ablation and served as controls. None of the patients received general anaesthesia for their procedures; however, patients were given sedation (15 of the AF patients and six of the non-AF patients) and analgesia (midazolam, morphine) as required if they were restless or in discomfort. Isoproterenol was given to provoke tachycardia if this was felt necessary. All patients were in sinus rhythm before and after their procedures.

Radiofrequency energy was used for ablation in all patients with procedural details described in Table 1. Conventional end-points of ablation were used: wide area circumferential pulmonary vein isolation for atrial fibrillation (without any linear lesions or targeting of complex 
Table 1 Patient characteristics and ablation procedure

\begin{tabular}{lllll}
\hline & Age (years) & Sex (M/F) & Duration (min) & Procedure \\
\hline AF & $59 \pm 11$ & $6 / 9$ & $149 \pm 46$ & Segmental pulmonary vein isolation \\
AVNRT & $48 \pm 15$ & $3 / 12$ & $106 \pm 37$ & Slow pathway modification \\
AVRT & $34 \pm 16$ & $10 / 5$ & $153 \pm 72$ & Accessory pathway ablation \\
Atrial Flutter & $64 \pm 9$ & $8 / 7$ & $105 \pm 37$ & Cavo-tricuspid ablation \\
Negative EP Study & $43 \pm 17$ & $4 / 11$ & $94 \pm 34$ & No ablation \\
\hline
\end{tabular}

fractionated electrograms); bidirectional block across the cavo-tricuspid isthmus for atrial flutter; evidence of slow pathway modification for AVNRT and loss of pre-excitation or non-inducibility for AVRT. There was no deliberate attempt to modify or extend the ablation lesions to target areas of dense autonomic innervation [5].

Procedures were defined as beginning at needle to skin and ending at catheter removal. All recordings were taken in the period between these events (i.e. intra-procedural). Sinus rhythm ECG recordings were taken for at least $5 \mathrm{~min}$ at the beginning and at the end of each procedure, with patients lying still on the operating table. The ECG recordings were stored on a Bard system (Bard EP, Lowell, MA, USA) for offline analysis. The ECG with the largest R wave amplitude was visually inspected to exclude ectopic beats before exporting segments for analysis.

Using the shortest duration that yielded valid HRV results from the study in normal volunteers, at least five 40-s ECG segments were exported at 1,000 Hz sampling frequency for HRV analysis to obtain average LF and HF values immediately before and after the procedure to ascertain if the expected changes in autonomic modulation was observed.

To prevent confounding by isoproterenol, we separately grouped patients who received isoproterenol during the procedure and those who did not. We performed multivariate analysis to establish whether patient age and procedure duration could contribute to the changes observed in patients who did not receive isoproterenol.

In a separate group of 14 patients undergoing ablation for AF, the LF and HF powers were calculated in a step-wise approach to see if ablation at any particular site was responsible for HRV changes observed. HRV indices were therefore measured at the following steps: baseline, after transseptal puncture, after right pulmonary vein circumferential ablation and after left pulmonary vein circumferential ablation. Seven patients underwent left-sided ablation before right-sided ablation and seven patients had right-sided ablation before left-sided ablation.

\subsection{Statistical analysis}

Data are expressed as mean \pm standard deviation. The power of each frequency band was logarithmically transformed to normalise the distribution [8]. Pre- and post-ablation LF and HF HRV values were then compared using the twotailed paired Student's $t$ test. A value of $p<0.05$ indicates statistical significance. The potential confounding effects of age and procedure duration on changes in HRV were analysed in multivariate linear regression analyses. All statistical tests were performed using Stata v10.1 (StataCorp LP, TX, USA).

\section{Results}

3.1 Short duration recordings used for heart rate variability analysis in healthy volunteers

Fifteen healthy volunteers with a mean age of $43 \pm 12$ years were studied under control conditions with continuous ECG monitoring for $20 \mathrm{~min}$. A 5-min ectopy-free recording segment was chosen and analysed in progressively shorter durations from $5 \mathrm{~min}$ down to $20 \mathrm{~s}$.

The HF power, representing respiratory sinus arrhythmia, which has a cycle time typically of 3-4 s, was shown to have no consistent shift in its mean value as the analysis window was shortened, although as can be seen from the graph (Fig. 1a), there was increased scatter when the recording duration was 30 or $20 \mathrm{~s}$.

The LF power, representing oscillations whose typical cycle length is of the order of $10 \mathrm{~s}$, showed no significant variability as the analysis window duration was shortened down to $40 \mathrm{~s}$. However, at window durations shorter than $40 \mathrm{~s}$, there was a significant difference and increased scatter in the mean LF values (Fig. 1b).

The intra-class correlations coefficients, calculated following variance components analyses were 0.80 for $\mathrm{HF}$ and 0.93 for LF, confirming that in these indices measured for different durations, within-subject variance was small compared with between-subject variance.

3.2 Short duration recordings used for heart rate variability analysis in patients undergoing tilt table testing

To validate this method, LF and HF HRV was calculated at baseline and immediately before onset of syncope using 
(a)
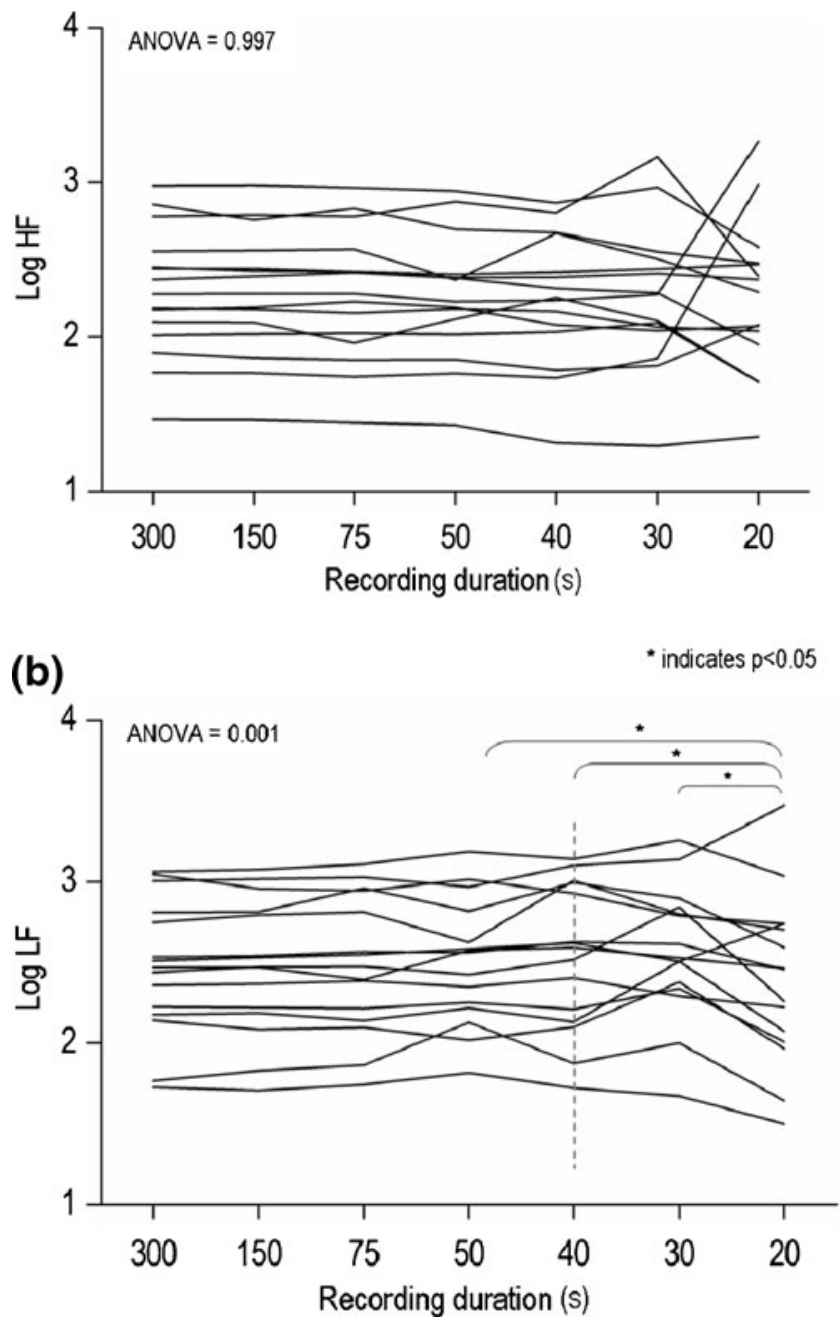

Fig. 1 Validation of short recording duration for HRV calculation. Log HF (a) and $\log$ LF (b) values plotted against duration of analysis in 15 healthy volunteers. The HF power showed no shift in mean value in all recording durations, although scatter increased at 30 and $20 \mathrm{~s}$ recording durations. The LF power showed no shift in mean value down to $40 \mathrm{~s}$. However, at window durations shorter than $40 \mathrm{~s}$, there was a significant difference $(p<0.05)$, and increased scatter in the mean LF values. Mean HRV indices for each recording duration compared by one-way ANOVA with Newman-Keuls correction for comparison between the means. $H F$ high frequency, $L F$ low frequency, $H R V$ heart rate variability

multiple $40 \mathrm{~s}$ windows in ten patients (mean age, 48 \pm 22 ) undergoing tilt table testing for vasovagal syncope. The LF and HF HRV, averaged from 40-s windows, were found to increase significantly immediately preceding syncope (LF: $341 \pm 311-$ $1,536 \pm 1,368 \mathrm{~ms}^{2}, p<0.01$; HF: $342 \pm 339-1,628 \pm 1,755 \mathrm{~ms}^{2}$, $p<0.05)$. HRV assessed from conventional 5-min windows behaved in the same way (LF: $251 \pm 242-735 \pm 440 \mathrm{~ms}^{2}, p<$ 0.01, HF: $\left.297 \pm 318-778 \pm 739 \mathrm{~ms}^{2}, p<0.05\right)$.

\subsection{Ablation procedures and success}

Radiofrequency ablation was deemed a primary success in all 60 patients undergoing ablation. None of the patients experienced any acute complication during the procedure.

3.4 Acute change in heart rate variability analysed using multiple 40 -s windows

The AF ablation procedure (which in no case involved isoproterenol use) significantly reduced LF by $67 \%$ and HF by $61 \%$ (example shown in left panel of Fig. 3 ). There was no change in the mean RR interval $(877 \pm 191-843 \pm$ $220 \mathrm{~ms}, \mathrm{p}=0.26$ ) or the LF/HF ratio.

The other EP procedures, in which no isoproterenol was used (15 atrial flutter, five AVNRT, nine AVRT, and five negative EP studies) had no effect on the mean RR interval, LF, HF or LF/HF values (Table 2, Fig. 2). An example of LF and HF values following ablation for atrial flutter is shown in the right panel of (Fig. 3).

The remaining procedures, which were performed with isoproterenol administration (ten AVNRT, six AVRT and ten negative EP studies), showed a reduction in the mean RR interval, accompanied by the expected reductions in LF and HF HRV and an increase in the LF/HF ratio (Table 2, Fig. 2) detected using the multiple 40-s window technique.

3.5 Intra-procedural changes in heart rate variability assessed in a step-wise approach

Fourteen patients with a mean age of $59 \pm 8$ years and a mean AF duration of $56 \pm 30$ months were studied for stepwise changes in HRV. In both groups, a significant reduction in both LF and HF HRV were only observed following right pulmonary vein, but not left pulmonary vein wide area circumferential ablation (Fig. 4).

3.6 The effect of patient age and procedure duration on heart rate variability changes

In order to determine whether procedure duration had an effect on the changes in HRV, we carried out a multivariate analysis to compare changes recorded in the AF ablation $(n=15)$ and the non-AF procedures in which no isoproterenol was used $(n=34)$ (Table 3 ). There was only a non-significant trend towards falling LF and HF after longer procedures. The effect of AF ablation is much larger, is clearly statistically significant, is equally evident in both LF and HF HRV, and persists even after adjusting for any effect of age or procedure duration. The unadjusted regression coefficients for the between group differences in log (LF) HRV and log 


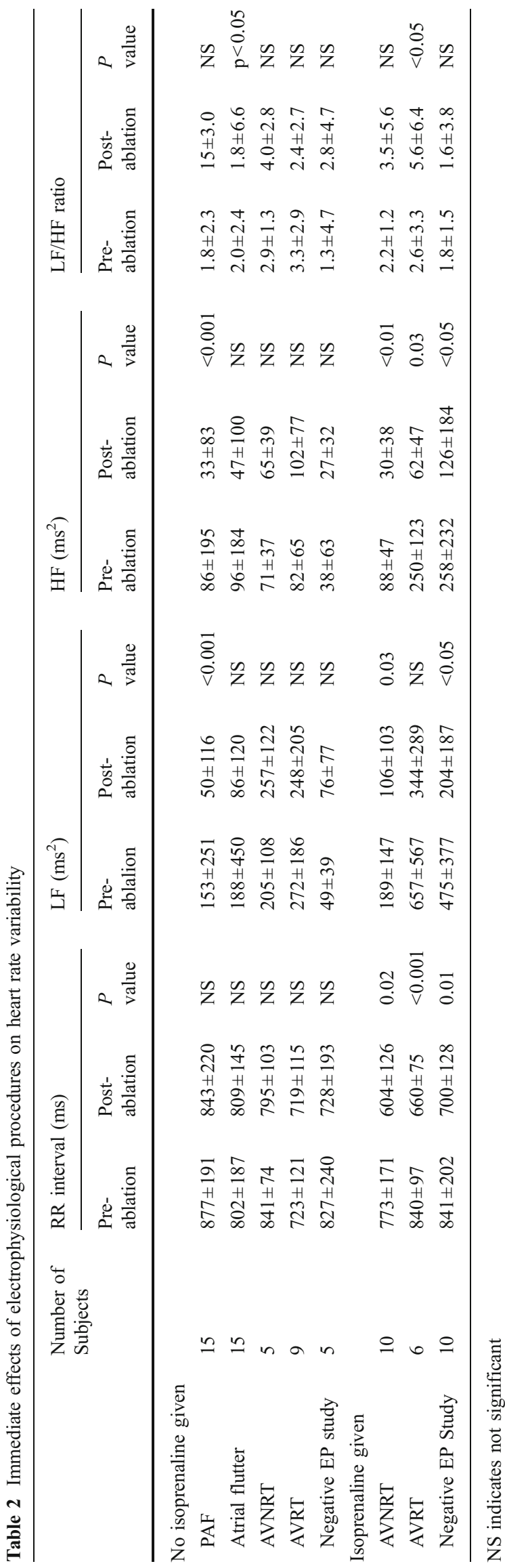

(HF) HRV were -0.61 (95\%CI: $-0.99,-0.22, p=0.003)$ and $-0.40(-0.73,-0.06, p=0.023)$, and following adjustment for age and procedure duration: $-0.51(-0.88,-0.14, p=0.008)$ and $-0.33(-0.61,-0.05, p=-0.021)$, respectively.

\section{Discussion}

This study presents and validates a technique for measuring HRV using shorter windows of time than are typically used. Averaging windows of $40 \mathrm{~s}$ (a duration that is easily visualised on a single screen during a procedure to establish that it is ectopic-free) gives data comparable to 5-min values which are conventionally recommended for ambulant outpatients undergoing routine HRV assessment, and readily detects intraprocedural autonomic changes within individuals.

Although HRV changes have been previously documented following AF ablation, the use of this technique intraprocedurally has, for the first time in humans, demonstrated that significant $\mathrm{HRV}$ reduction occurs following right, but not left-sided pulmonary vein wide area circumferential ablation.

\subsection{Real-time intra-procedural recordings: validity} and potential utility

We showed that average LF and HF indices of HRV remained stable with recording durations as brief as $40 \mathrm{~s}$ compared with $5 \mathrm{~min}$ recordings in healthy volunteers, despite the increase scatter seen with the 40 -s window. The five 40-s recordings used encompassed a total of $200 \mathrm{~s}$, which is approximately 20 cycles worth of the typical 10 -s cycles of LF oscillations and many more cycles of HF oscillations: from basic mathematical principles, this is adequate to quantify oscillatory amplitude.

This method of monitoring HRV reliably detected a significant increase in LF and HF HRV immediately preceding syncope in patients undergoing tilt table testing [13]. This short recording window identified HRV changes in patients undergoing catheter ablation, which were consistent with previous studies using longer term recordings $[11,14]$.

In addition, short-segment HRV measurements taken at sequential stages of an $\mathrm{AF}$ ablation procedure identified significant intraprocedural changes in HF and LF HRV following right-sided circumferential pulmonary vein ablation, but not left-sided circumferential ablation or transseptal puncture.

Our study shows that the principle of HRV analysis using multiple short-term windows is valid. Ectopy-free segments of this duration are easy to collect, and clearly the effect size is large enough to be detectable in such recordings. Thus short-term recordings may have potential value during or immediately after ablation procedures. 
RR interval (ms)

No Isoprenaline

AF $(n=15)$

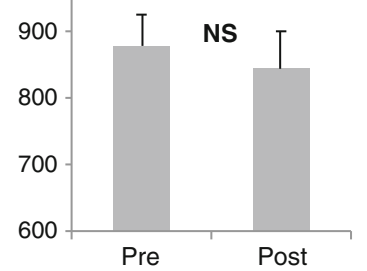

Non-AF

$(n=34)$

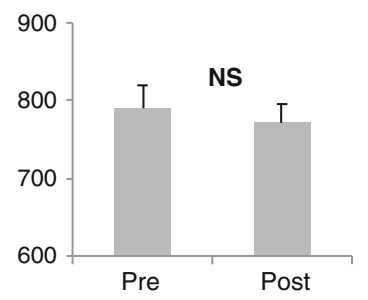

Isoprenaline

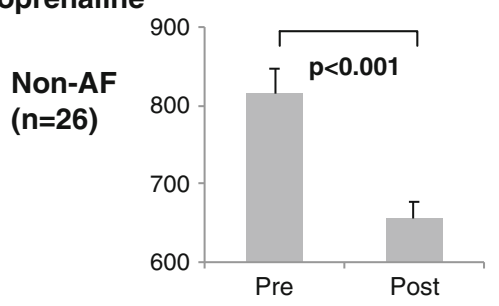

LF power $\left(\mathrm{ms}^{2}\right)$
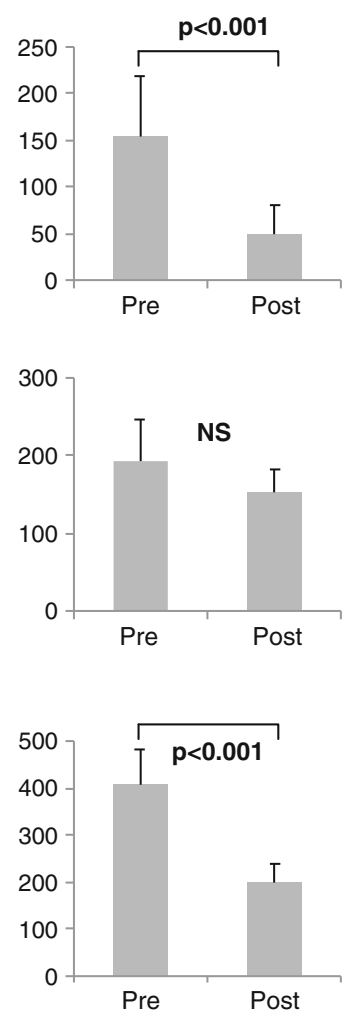

HF power $\left(\mathrm{ms}^{2}\right)$ $p<0.001$
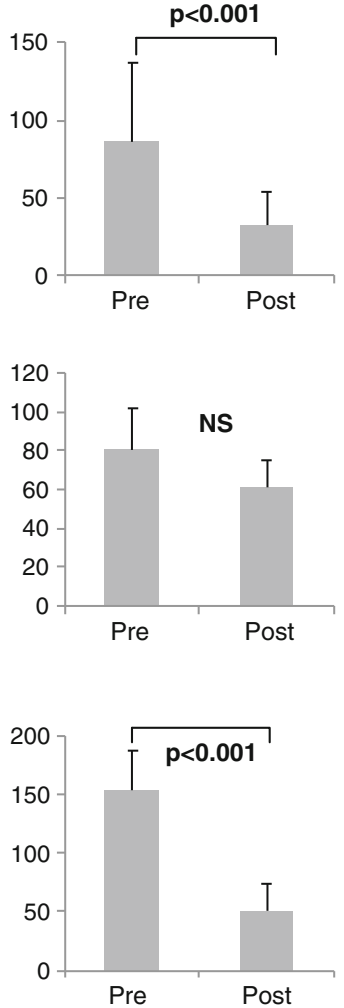

LF/HF Ratio
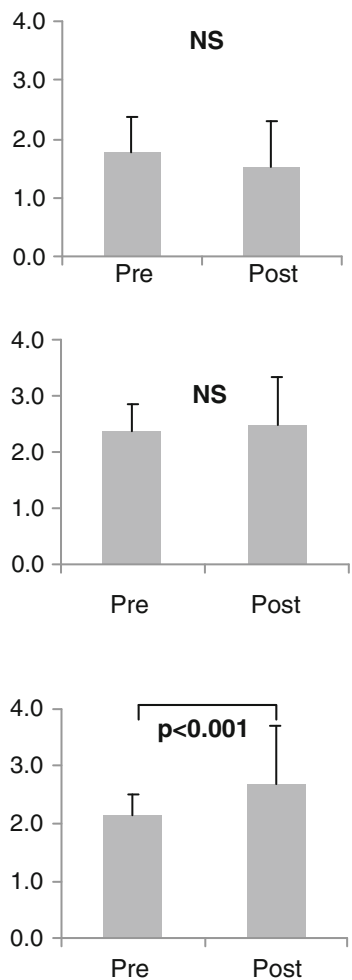

Fig. 2 Changes in RR interval and HRV indices after ablation. Non-AF includes patients having ablation for atrial flutter, atrioventricular nodal re-entry tachycardia, atrioventricular re-entrant tachycardia and negative electrophysiological studies. NS nonsignfiiciant

4.2 Specific immediate effect on heart rate variability of AF ablation

An important consideration when measuring changes in HRV is to ensure a constant RR interval, since any cause of reduced RR interval (i.e. sinus tachycardia) is necessarily accompanied by a smaller absolute amplitude of variability of the RR interval. We detected a significant reduction in HF and LF HRV following AF ablation, without a reduction in the $R R$ interval, suggesting that the reduction is due to modifications of autonomic nervous modulation.

Ablation of other arrhythmias did not affect HF or LF. However, if isoproterenol was given during the procedure, mean RR interval fell as expected, as did the HF and LF parameters of HRV.

\subsection{Autonomic modulation of the sinus node}

Autonomic modulation of the sinus node causes physiological oscillations in heart rate leading to LF and HF HRV [9]. Hou et al. [15] showed in animal studies that a significant amount of sympathetic and parasympathetic input to the sinus node traverses the right anterior ganglionated plexus located in the antral region of the right superior pulmonary vein. We postulate that in humans, a similarly sited ganglionated plexus is responsible for most of the nervous traffic to the sinus node and in turn can influence LF and HF variations in the heart rate. Our study showed HRV reduction occurred only following ablation around the right-sided pulmonary veins, which could conceivably disrupt autonomic nervous inputs into the sinus node.

\subsection{Implications for autonomic ablation}

Advances in understanding of functional cardiac neuroanatomy [16, 17] have led to development of autonomic ablation procedures that have been performed in humans in an attempt to treat cardiac arrhythmias including AF $[3,4,6,8]$ and neurocardiogenic syncope [18]. Autonomic ablation has been performed based on fixed anatomical landmarks [6], identification of distinct spectral patterns by fast Fourier transforms of endocardial signals [18], and identification and ablation of sites guided by high frequency stimulation [3, 4, 7] resulting in vagal responses. However, these methods do 

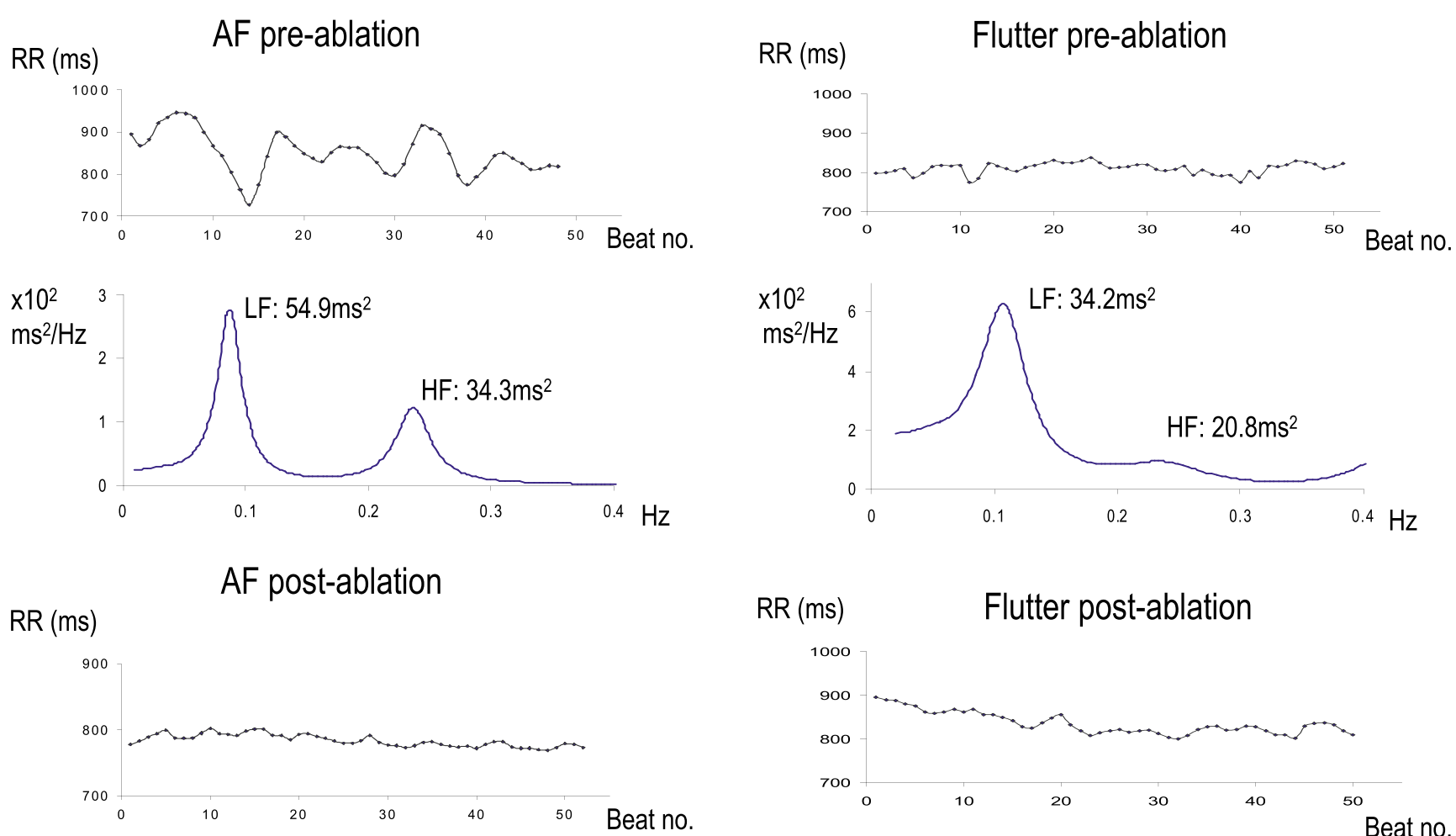

$\mathrm{RR}(\mathrm{ms}) \quad$ Flutter post-ablation
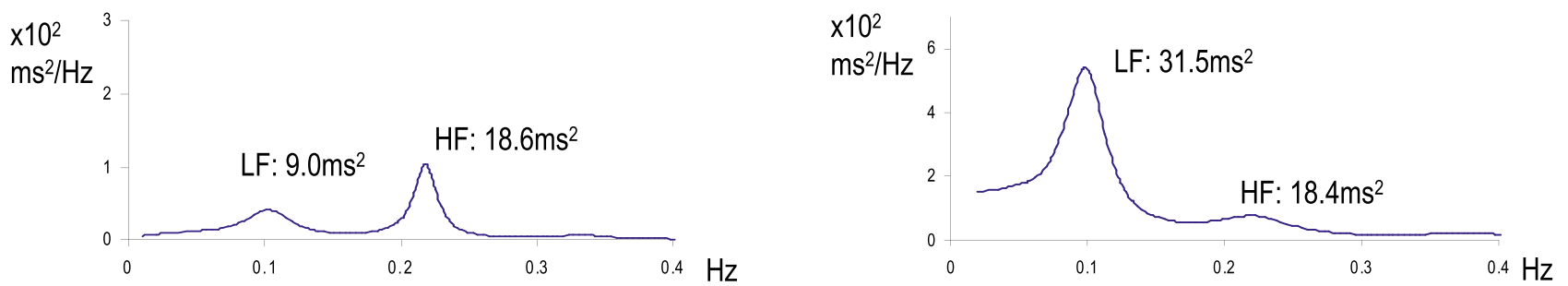

Fig. 3 Power spectral density and RR tachogram plots pre- and post-ablation power spectral density and RR tachogram plots for example patients in $\mathrm{AF}$ and atrial flutter

not provide a quantifiable measure of autonomic modification. Real-time measurement of HRV has the potential to be developed into a tool to assess intraprocedural autonomic modification.

\subsection{Limitations}

Healthy volunteers, typical of the general population in which conventional HRV measurements were developed, were used in the validation arm of this study to ascertain the minimum recording period that still yielded valid HRV results. In such subjects, ectopy-free ECG segments are easy to obtain. This is not always the case in patients undergoing catheter ablation, making a reason to study the usability of shorter (40 s) recordings for which ectopy-free segments are more easily recorded. Detectable changes in
HRV in such short recordings increase the potential practical clinical value of the findings during ablation procedures.

The changes in HRV during AF ablation appear to be related only to right-sided circumferential pulmonary vein ablation. There may, however, by important effects of ablation of autonomic ganglia in other regions of the heart (including adjacent to the left pulmonary veins), which are not reflected by HRV changes, but which might still affect ablation outcomes.

These findings may stimulate further development into acute real-time longer recordings, which would give greater confidence in the values obtained. We are currently developing a suitable processing system, contained within a "black box" that will permit on-line processing of the data to obtain intra-procedural HRV measurements during 
7 patients who had left before right pulmonary vein isolation.

7 patients who had right before left pulmonary vein isolation.
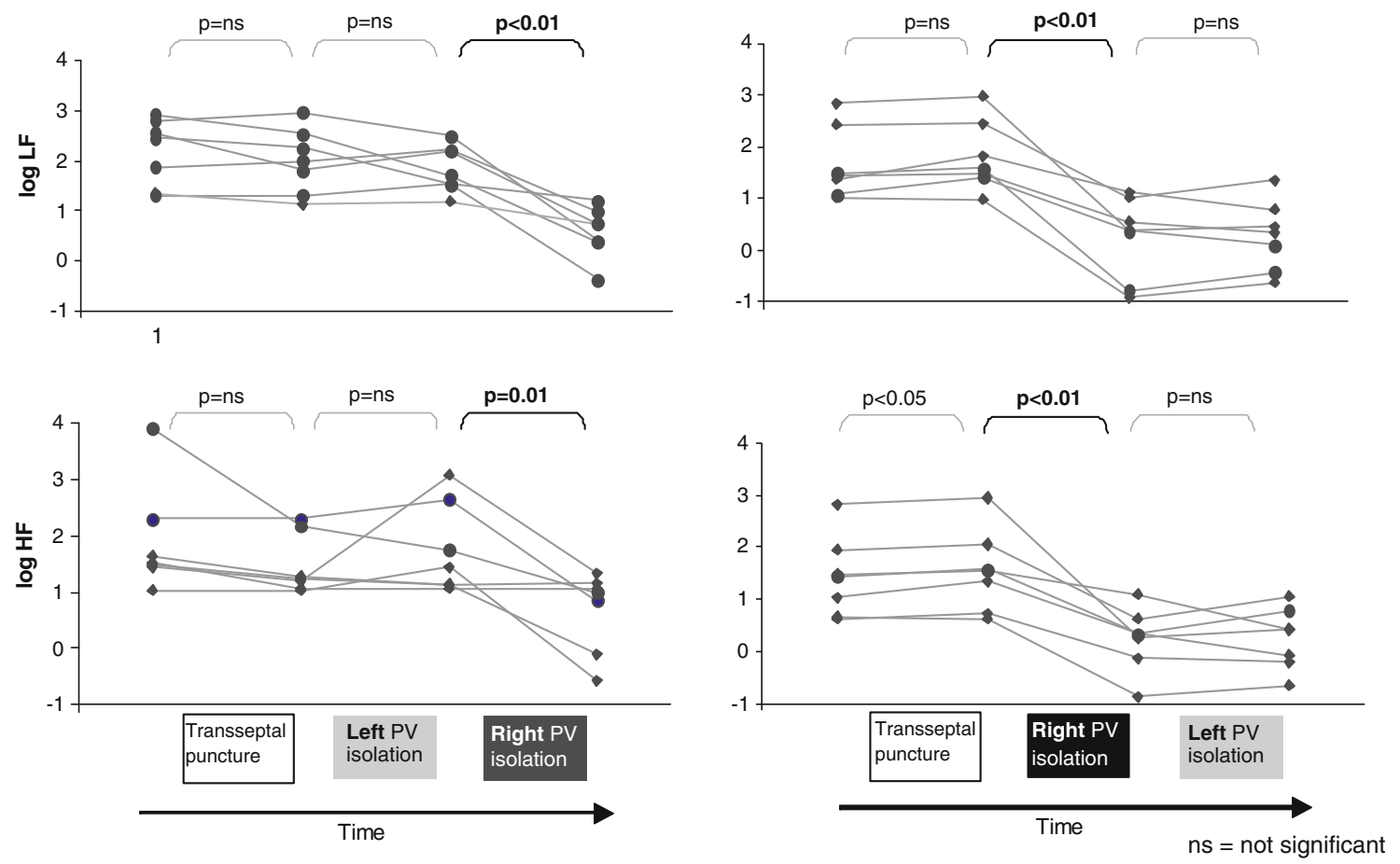

Fig. 4 Changes in HRV indices measured in a step-wise fashion Stepwise HRV changes in 14 patients. Seven patients had left before right pulmonary vein ablation and seven patients had right before left pulmonary vein wide area circumferential ablation. LF and HF HRV indices were measured in a step-wise fashion at each stage of the

procedure, including: baseline, after transseptal puncture, after leftsided circumferential PV isolation and after right-sided circumferential $\mathrm{PV}$ isolation. $H F$ high frequency, $L F$ low frequency, $H R V$ heart rate variability, $P V$ pulmonary veins

understanding of autonomic mechanisms of arrhythmias, which may ultimately lead to development of clinically relevant autonomic ablation procedures. ments will facilitate further studies to improve our

Table 3 Univariate and multivariate regression coefficients

Regression coefficient

Dependent variable $=$ delta $\log \mathrm{LF}$

Univariate

Age

Procedure duration

AF ablation vs. non-AF ablation

Mutivariate

Dependent variable $=$ delta $\log \mathrm{HF}$

Univariate

Age

Procedure duration

$\mathrm{AF}$ ablation vs. non-AF ablation

Multivariate

$\mathrm{AF}$ ablation vs. non-AF ablation adjusted for age and procedure
$\mathrm{AF}$ ablation vs. non-AF ablation adjusted for age and procedure

$\begin{array}{lll}-0.003 & 0.005 & 0.62 \\ -0.005 & 0.002 & 0.061 \\ -0.608 & 0.191 & 0.003 \\ & & \\ -0.512 & 0.183 & 0.008 \\ & & \\ 0.000 & 0.005 & 0.93 \\ -0.003 & 0.002 & 0.137 \\ -0.395 & 0.169 & 0.023 \\ -0.333 & & \\ & 0.139 & 0.021\end{array}$

Dependent variables: delta $\log \mathrm{LF}$ and delta $\log \mathrm{HF}$ in participants who did not receive isoproterenol $(n=49)$ 


\section{Conclusions}

Multiple windows as short as $40 \mathrm{~s}$ give valid measurements of mean HF and LF HRV. Such measurements are easily obtainable during routine EP procedures.

Left atrial ablation around the right-sided pulmonary veins is unique in producing reductions in LF and HF HRV, which is immediately detectable from multiple 40-s recordings. The ability to detect LF and HF HRV modification may, in the future, permit valuable intra-procedural measurements, ultimately able to establish end-point markers for adjunctive autonomic ablation procedures.

Acknowledgments We are grateful for the technical assistance from Gabrielle Appleton, Robert J Edwards, Anthony J Mason and Keith Willson. Dr P B Lim, Dr D P Francis and Dr M Koa-Wing are supported by the British Heart Foundation grants FS/06/089, FS/04/ 079 and PG/04/126. This work was supported by the NIHR Biomedical Research Scheme.

Financial disclosures Drs P B Lim, M Koa-Wing and D P Francis are supported by the British Heart Foundation grants FS/06/089, FS/ 04/079 and $\mathrm{PG} / 04 / 126$, respectively.

Open Access This article is distributed under the terms of the Creative Commons Attribution Noncommercial License which permits any noncommercial use, distribution, and reproduction in any medium, provided the original author(s) and source are credited.

\section{References}

1. Po, S. S., Scherlag, B. J., Yamanashi, W. S., Edwards, J., Zhou, J., $\mathrm{Wu}, \mathrm{R}$., et al. (2006). Experimental model for paroxysmal atrial fibrillation arising at the pulmonary vein-atrial junctions. Heart Rhythm, 3(2), 201-208.

2. Zhou, J., Scherlag, B. J., Edwards, J., Jackman, W. M., Lazzara, R., \& Po, S. S. (2007). Gradients of atrial refractoriness and inducibility of atrial fibrillation due to stimulation of ganglionated plexi. Journal of Cardiovascular Electrophysiology, 18(1), 83-90.

3. Scherlag, B. J., Nakagawa, H., Jackman, W. M., Yamanashi, W. S., Patterson, E., Po, S., et al. (2005). Electrical stimulation to identify neural elements on the heart: their role in atrial fibrillation. Journal of Interventional Cardiac Electrophysiology, 13(Suppl 1), 37-42.

4. Scanavacca, M., Pisani, C. F., Hachul, D., Lara, S., Hardy, C., Darrieux, F., et al. (2006). Selective atrial vagal denervation guided by evoked vagal reflex to treat patients with paroxysmal atrial fibrillation. Circulation, 114(9), 876-885.

5. Armour, J. A., Murphy, D. A., Yuan, B. X., Macdonald, S., \& Hopkins, D. A. (1997). Gross and microscopic anatomy of the human intrinsic cardiac nervous system. The Anatomical Record, 247(2), 289-298.

6. Pokushalov, E. (2008). The role of autonomic denervation during catheter ablation of atrial fibrillation. Current Opinion in Cardiology, 23(1), 55-59.

7. Lemery, R. (2006). How to perform ablation of the parasympathetic ganglia of the left atrium. Heart Rhythm, 3(10), 1237-1239.

8. Pappone, C., Santinelli, V., Manguso, F., Vicedomini, G., Gugliotta, F., Augello, G., et al. (2004). Pulmonary vein denervation enhances long-term benefit after circumferential ablation for paroxysmal atrial fibrillation. Circulation, 109(3), 327-334.

9. Task Force of the European Society of Cardiology and the North American Society of Pacing and Electrophysiology. (1996). Heart rate variability. Standards of measurement, physiological interpretation, and clinical use. European Heart Journal, 17(3), 354-381.

10. Redfearn, D. P., Skanes, A. C., Gula, L. J., Griffith, M. J., Marshall, H. J., Stafford, P. J., et al. (2007). Noninvasive assessment of atrial substrate change after wide area circumferential ablation: a comparison with segmental pulmonary vein isolation. Annals of Noninvasive Electrocardiology, 12(4), 329337.

11. Bauer, A., Deisenhofer, I., Schneider, R., Zrenner, B., Barthel, P., Karch, M., et al. (2006). Effects of circumferential or segmental pulmonary vein ablation for paroxysmal atrial fibrillation on cardiac autonomic function. Heart Rhythm, 3(12), 1428-1435.

12. Davies, L. C., Francis, D., Jurak, P., Kara, T., Piepoli, M., \& Coats, A. J. (1999). Reproducibility of methods for assessing baroreflex sensitivity in normal controls and in patients with chronic heart failure. Clinical Science (London), 97(4), 515522

13. Kochiadakis, G. E., Orfanakis, A., Chryssostomakis, S. I., Manios, E. G., Kounali, D. K., \& Vardas, P. E. (1997). Autonomic nervous system activity during tilt testing in syncopal patients, estimated by power spectral analysis of heart rate variability. Pacing and Clinical Electrophysiology, 20 (5 Pt 1), 1332-1341.

14. Markowitz, S. M., Christini, D. J., Stein, K. M., Mittal, S., Iwai, S., Slotwiner, D. J., et al. (2004). Time course and predictors of autonomic dysfunction after ablation of the slow atrioventricular nodal pathway. Pacing and Clinical Electrophysiology, 27(12), $1638-1643$.

15. Hou, Y., Scherlag, B. J., Lin, J., Zhou, J., Song, J., Zhang, Y., et al. (2007). Interactive atrial neural network: determining the connections between ganglionated plexi. Heart Rhythm, 4(1), 56-63.

16. Ardell, J. L., \& Randall, W. C. (1986). Selective vagal innervation of sinoatrial and atrioventricular nodes in canine heart. The American Journal of Physiology, 251(4 Pt 2), H764-H773.

17. Randall, W. C., Rinkema, L. E., \& Jones, S. B. (1984). Local epicardial chemical ablation of vagal input to sino-trial and atrioventricular regions of the canine heart. Journal of the Autonomic Nervous System, 11(2), 145-159.

18. Pachon, J. C., Pachon, E. I., Pachon, J. C., Lobo, T. J., Pachon, M. Z., Vargas, R. N., et al. (2005). "Cardioneuroablation"-new treatment for neurocardiogenic syncope, functional $\mathrm{AV}$ block and sinus dysfunction using catheter RF-ablation. Europace, 7(1), 1-13. 